\title{
Cellulose Acetate Based Material with Antibacterial Properties Created by Supercritical Solvent Impregnation
}

\author{
Stoja Milovanovic, ${ }^{1}$ Tijana Adamovic, ${ }^{1}$ Ksenija Aksentijevic, ${ }^{2}$ \\ Dusan Misic, ${ }^{2}$ Jasna Ivanovic, ${ }^{1}$ and Irena Zizovic ${ }^{1}$ \\ ${ }^{1}$ University of Belgrade Faculty of Technology and Metallurgy, Karnegijeva 4, 11000 Belgrade, Serbia \\ ${ }^{2}$ University of Belgrade Faculty of Veterinary Medicine, Bulevar Oslobođenja 18, 11000 Belgrade, Serbia \\ Correspondence should be addressed to Stoja Milovanovic; smilovanovic@tmf.bg.ac.rs
}

Received 29 March 2017; Accepted 1 June 2017; Published 4 July 2017

Academic Editor: Bernabé L. Rivas

Copyright (C) 2017 Stoja Milovanovic et al. This is an open access article distributed under the Creative Commons Attribution License, which permits unrestricted use, distribution, and reproduction in any medium, provided the original work is properly cited.

\begin{abstract}
Supercritical $\mathrm{CO}_{2}$ was used as a green solvent and impregnation medium for loading cellulose acetate beads with carvacrol in order to obtain a biomaterial with antibacterial properties. Supercritical solvent impregnation was performed in a high-pressure view cell at temperature of $50^{\circ} \mathrm{C}$ and pressures of 10,21 , and $30 \mathrm{MPa}$ with the processing time ranging from 2 to $18 \mathrm{~h}$. The rate of impregnation increased with the pressure increase. However, maximum impregnation yield (round 60\%) was not affected by the pressure applied. Selected samples of the impregnated cellulose acetate containing 6-60\% of carvacrol were proven to have considerable antibacterial effect against Gram-positive and Gram-negative bacterial strains including methicillin-resistant Staphylococcus aureus which causes severe infections in humans and animals. In addition, cellulose acetate beads containing $6.0-33.6 \%$ of carvacrol were shown to have a porous structure with submicron pores which is of interest for the controlled delivery applications.
\end{abstract}

\section{Introduction}

Process of supercritical solvent impregnation (SSI) implies dissolution of an additive in supercritical fluid and its subsequent incorporation into a polymer matrix $[1,2]$ which leads to formation of an added-value polymeric material. Reportedly, supercritical carbon dioxide $\left(\mathrm{scCO}_{2}\right)$ can be used to load polymeric carriers with antimicrobial substances [212], vitamins [13], drugs [14, 15], dyes [16, 17], and so on. There are two distinctive mechanisms of SSI. The first one involves simple deposition of an additive which is soluble in supercritical fluid into the polymer matrix due to decompression of the system. The second one involves impregnation of additives that have higher affinity towards the polymer and then to the supercritical fluid (due to chemical interaction between the additive and polymer) $[1,2]$. SSI is advantageous over conventional impregnation methods because it excludes the use of organic solvents as well as the drying step. Also, impregnation using $\mathrm{scCO}_{2}$ can be conducted at relatively low temperatures due to the low critical temperature of $\mathrm{CO}_{2}\left(31.1^{\circ} \mathrm{C}\right)$, which allows the use of thermolabile additives and heat sensitive polymers. Finally, unique and tuneable transport properties of supercritical fluids $[1,13]$ enable fast and controlled penetration and dispersion of an additive into the carrier [14]. Beside environmental benefits reflected in avoidance of hazardous solvents usage or waste water generation, application of SSI provides products free of solvent residues. $\mathrm{ScCO}_{2}$ is simply eliminated and recovered from the final products by depressurization. Another benefit of $\mathrm{scCO}_{2}$ use is that it dissolves in most of polymers causing their swelling [6]. This facilitates incorporation of substances dissolved in a supercritical fluid into the polymer matrix. Acting like a molecular lubricant $\mathrm{scCO}_{2}$ affects thermal properties of polymeric material and can be also used to adjust morphological characteristics of the final product $[1$, 14]. In summary, SSI provides controlled product quality and safety as well as energy and time saving.

Outbreak of panresistant bacterial strains led to a growing demand for materials with antibacterial properties containing active substances other than antibiotics. If the material is to be applied for biomedical purposes, another demand may arise: to be biodegradable. Natural compounds and 
plant extracts hold great promise as alternative antimicrobial agents since there has been no evidence of their contribution to further bacterial resistance [18-20]. Essential oils from herbs belonging to Lamiaceae family are widely used in food, pharmaceutical, and cosmetic industries [21]. Carvacrol, a phenolic monoterpenoid abundantly present in essential oils of herbs belonging to genus Origanum [21], was selected for this study due to its well-known antibacterial and antioxidant properties [11, 22-24] as well as its approved status by the FDA as a safe food additive [10, 23]. Promising prospective of carvacrol applications has stimulated researchers to study its incorporation into different polymers (e.g., cyclodextrin, cellulose, gelatin, and chitosan) for different purposes $[10,11$, $23,24]$. Cellulose acetate (CA) holds a great promise for food, pharmaceutical, and medicinal applications since it is stable, biocompatible, bioresorbable, and derived from cellulose, the world's most abundant biopolymer [7, 25-27]. Its good properties stimulated research focused on the generation of CA structures (membranes, foams, microparticles etc.) that contain bioactive compounds $[4,5,14,28-30]$. These CA structures can be produced with methods like solvent casting, electrospinning, supercritical phase separation, and so on and used for controlled delivery of additives [14, 28-30]. CA foams with scopolamine and CA films with pepper essential oil produced by solvent casting method can be used for skin treatment [28] and as active food packaging [29], respectively. CA membranes produced by electrospinning and subsequent immersion in amoxicillin solution can have gastrointestinal application [30]. CA microparticles, nanofibrous networks, and cellular membrane with nonsteroidal anti-inflammatory drug ibuprofen produced by supercritical fluid technology were reported for tissue engineering applications [14].

Present study was aimed at investigating feasibility of SSI for impregnation of CA beads with carvacrol as well as antibacterial activity of the obtained material. Applied singlestep batch SSI process involved dissolution of carvacrol in $\mathrm{scCO}_{2}$ and its incorporation into CA. The effect of operating pressure on impregnation yield and morphology of the polymer substrate was discussed in detail. Antibacterial activity of CA impregnated with carvacrol was investigated against sixteen bacterial strains, including methicillin-resistant Staphylococcus aureus (MRSA), which are relevant for potential applications of the developed added-value material in the food industry and biomedicine.

\section{Materials and Methods}

2.1. Materials. Carvacrol (purity $>99 \%$ ) was supplied by Sigma-Aldrich (Germany). Pharmaceutical grade cellulose acetate beads (CA-320S NF/EP) with acetyl content $32.0 \%$ were generous donation from Eastman (Poland). Commercial $\mathrm{CO}_{2}$ (purity 99\%) was supplied by Messer-Tehnogas (Serbia).

\subsection{Methods}

2.2.1. Supercritical Impregnation of Cellulose Acetate with Carvacrol. Supercritical impregnation of CA with carvacrol was performed using the static method in a high-pressure view cell (Eurotechnica GmbH, Germany) presented in Figure 1 and previously described in detail $[3,5]$. Carvacrol was placed on the bottom of view cell in a glass container with a fine mesh on its top to avoid possible splashing during decompression. CA beads were placed in a porous basket above the carvacrol. Liquid $\mathrm{CO}_{2}$ from a siphon type cylinder was precooled in a cryostat $(\mathrm{C})$ and introduced into the view cell previously heated to $50^{\circ} \mathrm{C}$. Pressurization of the system to the working pressure of 10,21 , or $30 \mathrm{MPa}$ using a highpressure pump (P) for liquids (Milton Roy, France) followed. The impregnation time was varied from 2 to $18 \mathrm{~h}$. Initial mass ratio of carvacrol: CA was $12: 1$ in all the experiments, except for the sample impregnated at $30 \mathrm{MPa}$ for $18 \mathrm{~h}$ when the ratio of $72: 1$ was used in order to provide carvacrol in excess like in other experiments. After each experiment, the $\mathrm{CO}_{2}$ was released from the vessel at the rate of $0.3 \mathrm{MPa} / \mathrm{min}$.

Impregnated mass of carvacrol $\left(m_{\text {carv }}\right)$ was determined gravimetrically by measuring the polymer mass at the beginning of the process and after the impregnation. Impregnation yield $(I)$ of carvacrol was calculated according to the equation

$$
I=\frac{m_{\mathrm{carv}}}{m_{\mathrm{CA}}+m_{\mathrm{carv}}} \cdot 100 \%,
$$

where $m_{\mathrm{CA}}$ is the mass of CA at the beginning of the process.

2.2.2. Characterization of the Samples. Field emission scanning electron microscopy (SEM, Mira3 Tescan) of the CA beads was used to determine CA morphology before and after supercritical impregnation of carvacrol. The samples were coated with a thin layer of $\mathrm{Au} / \mathrm{Pd}(85 / 15)$ prior to the analysis.

2.2.3. Antibacterial Activity. The agar disk diffusion test was used as a qualitative assessment of antibacterial activity of CA impregnated with carvacrol against selected Grampositive and Gram-negative bacterial strains: Acinetobacter sp. (GA11), Bacillus anthracis, Bacillus cereus, Bacillus subtilis, Corynebacterium sp., Escherichia coli, Klebsiella pneumoniae (GA15), Listeria ivanovii ATCC19119, Listeria monocytogenes ATCC19111, Rhodococcus equi, Salmonella Enteritidis, Streptococcus pyogenes, Streptococcus pneumoniae, and three clinical isolates of Methicillin-resistant Staphylococcus aureus (MRSA). Neat CA samples were used as negative control. All investigated bacterial strains were isolated from clinical specimen delivered to routine microbiological examination except the strains from the American Type Culture Collection (ATCC). Conventional bacteriological methods were applied for the isolation of bacteria with the use of MacConkey agar (Becton Dickinson), Columbia 5\% sheep blood agar (BioMérieux), Rappaport-Vassiliadis semisolid agar (Becton Dickinson), buffered peptone water (Difco), and XLT4 agar (Becton Dickinson). For identification of isolated bacteria identification systems API ID32 (BioMérieux), BBL Crystal Gram-positive ID system, and BBL Crystal Enteric/Nonfermenter ID system (Becton Dickinson) were used. For serological typisation of Salmonella specific diagnostic sera were used in slide agglutination test (Statens Serum Institute, Denmark). For the detection of MRSA strains, cefoxitin discs were used (Becton Dickinson) in 


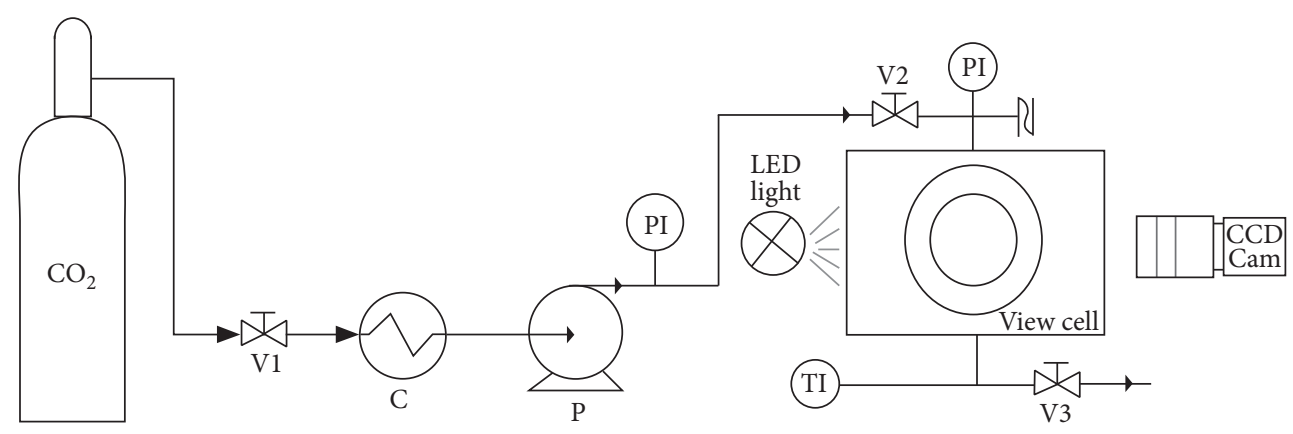

Figure 1: Schematic presentation of the view cell.

routine disc diffusion investigations, and confirmation was achieved by detecting mecA gene using PCR according to previously described protocols [31].

For the purpose of investigation of antibacterial activity of the CA samples (pure CA and impregnated with carvacrol), disc diffusion method recommended by Clinical and Laboratory Standards Institute (CLSI) and the European Committee on Antimicrobial Susceptibility Testing (EUCAST) was used. The desired inoculums (density of approximately $1-2 \times$ $108 \mathrm{CFU} / \mathrm{mL}$ ) were achieved by preparing the suspension of bacteria with the density equal to McFarland standard 0.5 (Becton Dickinson). The investigation was conducted on Mueller Hinton agar (BioMérieux, France) and Mueller Hinton agar with $6 \%$ sheep blood (BioMérieux, France). The only modification was that instead of antibiotics cellulose acetate with carvacrol was used. The results were read by measuring the diameter of the inhibition zones after the incubation period of $18-24$ hours at $37^{\circ} \mathrm{C}$. No interpretive categorization was used (susceptible, resistant) because there are no standards for this kind of investigation.

\section{Results and Discussion}

3.1. Supercritical Impregnation of Cellulose Acetate with Carvacrol. Amount of impregnated active substance and porous structure of the substrate (carrier) are of vital importance for controlled drug delivery and its efficiency against target bacterial strains. Amount of impregnated additive depends on complex interactions of ternary system (additivesupercritical fluid-polymer) which are affected by $\mathrm{CO}_{2}$ pressure, temperature, nature of polymer, and time of its exposure to supercritical fluid $[4,7,9]$. Previous studies demonstrated that pure $\mathrm{scCO}_{2}$ had negligible effect on CA morphology and thermal properties $[5,8]$. Therefore, manipulation of pressure and time in the SSI of CA with carvacrol was aimed at setting optimal operating conditions regarding mass transfer and structural changes of $\mathrm{CA}$ in the presence of $\mathrm{scCO}_{2}+$ carvacrol. Operating pressure $(10,21$ and $30 \mathrm{MPa})$ and temperature $\left(50^{\circ} \mathrm{C}\right)$ conditions for the impregnation process were chosen on the basis of previous report on carvacrol solubility in $\mathrm{CO}_{2}$ [21]. Obtained impregnation yields in the SSI process are presented in Table 1.

All the impregnation curves followed linear regime within the first few hours indicating fast impregnation (Figure 2). Steeper slope of impregnation curve in the linear

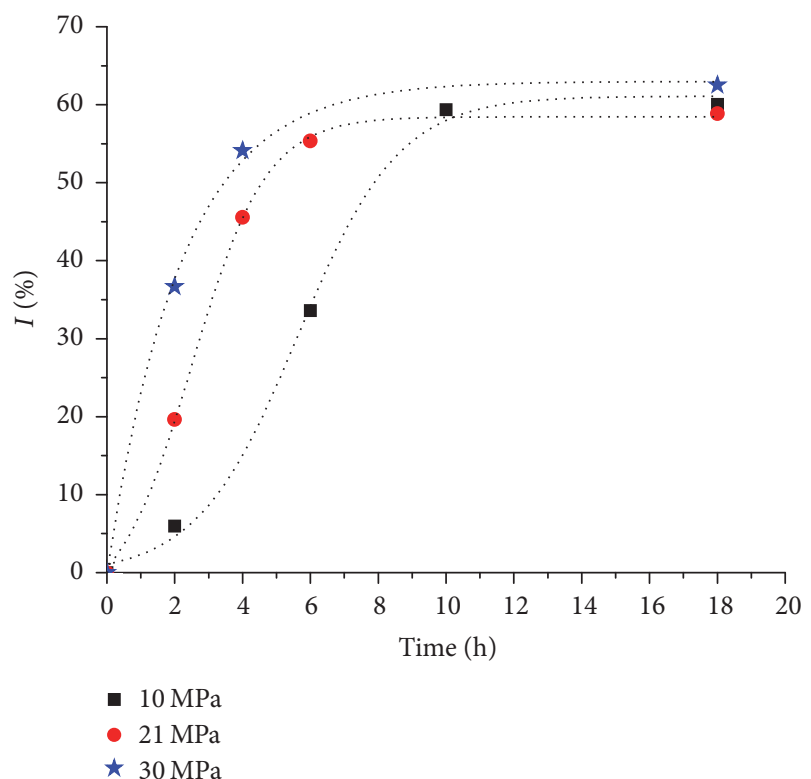

Figure 2: Change in carvacrol impregnation yield with time (the lines are drawn to guide the eye).

regime indicated faster impregnation rate at higher pressures. This could be result of increased diffusivity in solid matrix and higher carvacrol solubility [21] in the $\mathrm{scCO}_{2}$ which provide a larger driving force for the impregnation [4]. Similar phenomenon was shown for the SSI of cotton gauze and CA with thymol $[3,4]$. After the period of fast impregnation in the first few hours of the SSI, gradual decrease of the impregnation rate and equalization of the impregnation yield with the loading capacity of carvacrol in CA $(\sim 60 \%)$ followed. The maximum impregnation yield that corresponds to the loading capacity of the carrier was found to be around $60 \%$ regardless of the applied pressures, whereby the time $\left(t_{\operatorname{Imp}}^{\max }\right)$ for reaching it decreased with the pressure increase (Figure 2). Similar phenomenon was also observed for the SSI of CA with thymol where the time needed for achieving maximum impregnation yield $(\sim 70 \%)$ at $10 \mathrm{MPa}$ was almost three times longer ( $45 \mathrm{~h})$ compared the time needed at $20 \mathrm{MPa}(16 \mathrm{~h})$ [4].

For $t \leq t_{\mathrm{Imp}}^{\max }$ increase in pressure resulted in the increased impregnation yield of carvacrol (Figure 2). Namely, impregnation yield of carvacrol after $2 \mathrm{~h}$ of the SSI was reported to be $6.0 \%$ at $10 \mathrm{MPa}, 19.7 \%$ at $21 \mathrm{MPa}$, and $36.7 \%$ at $30 \mathrm{MPa}$ 
TABLE 1: Results of carvacrol impregnation into CA.

\begin{tabular}{lcccccccccc}
\hline $\begin{array}{l}\text { Pressure (MPa) } \\
\text { Time (h) }\end{array}$ & 2 & 6 & 10 & 18 & 2 & 4 & 6 & 18 & 2 & 4 \\
\hline$I(\%)$ & 6.0 & 33.6 & 59.4 & 60.0 & 19.7 & 45.6 & 55.4 & 58.9 & 36.7 & 54.1 \\
\hline
\end{tabular}

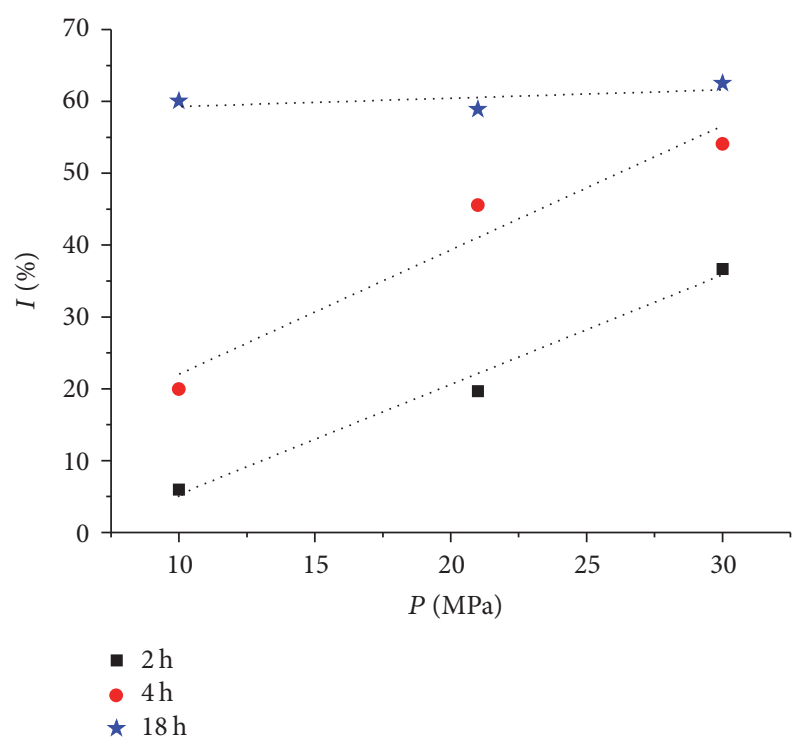

FIGURE 3: Change in carvacrol impregnation yield with pressure (the lines are drawn to guide the eye).

(Figure 3). This can be explained by the reported carvacrol solubility in $\mathrm{scCO}_{2}$ increase with pressure from $7.3 \mathrm{kmol} / \mathrm{mol}$ $(10 \mathrm{MPa})$ to $61.2 \mathrm{kmol} / \mathrm{mol}(21 \mathrm{MPa})$ and $178.2 \mathrm{kmol} / \mathrm{mol}$ (30 MPa) [21]. Increased carvacrol solubility with the operating pressure does not necessarily imply the impregnation yield increase. When there is a sufficient amount of an impregnating substance in a polymer $+\mathrm{scCO}_{2}$ system to achieve saturation at given operating conditions, affinity of the impregnating substance to the polymer phase decreases (as driving force for impregnation ceases) $[6,8]$. At the same time, affinity of the impregnating substance may be higher towards the supercritical fluid than to the polymer which may lead to the decrease in impregnation yield [6].

Some of previous reports on $\mathrm{CO}_{2}$-assisted impregnation of additives into CA present products with different end applications. Mallepally and coworkers [7] produced controlled topical oxygen delivery device by impregnation of CA with hydrogen peroxide at pressure of $8.3 \mathrm{MPa}$ and temperatures from 25 to $45^{\circ} \mathrm{C}$ during $1 \mathrm{~h}$ with a maximum impregnation yield of $25 \%$. Shen and coworkers [8] impregnated CA with vanillin and L-menthol at pressures from 5.2 to $17.6 \mathrm{MPa}$ and temperatures from 20 to $50^{\circ} \mathrm{C}$ during $2 \mathrm{~h}$ with impregnation yields up to $20 \%$ for possible application in the food industry.

3.2. Morphology of Cellulose Acetate Samples. Appearance of neat and CA beads impregnated with various amounts of carvacrol was evidently different (Figure 4). Neat CA sample was denoted as control (C). CA sample with the lowest

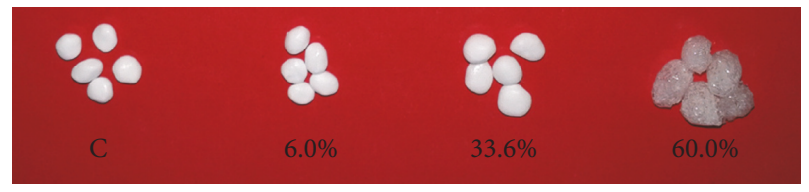

FIGURE 4: Effect of carvacrol loads on the swelling and agglomeration of CA beads.

impregnation yield (6.0\%) had the appearance similar to the control sample. Loading of higher percentage of carvacrol ( $35 \%)$ into CA induced swelling of the substrate. Extensive swelling of CA beads accompanied with agglutination was characteristic for the samples with impregnation yield above $55 \%$. Samples of CA impregnated with $6.0 \%, 33.6 \%$, and $60.0 \%$ of carvacrol (Figure 4 ) were used as representatives for further SEM and antibacterial tests.

Morphological changes in the selected impregnated samples of CA were confirmed by the SEM analysis. SEM images of surface and cross section of the control and CA samples loaded with different amounts of carvacrol are presented in Figure 5. Control sample had unevenly bumpy surface without visible pores while inner structure was shown to be highly porous with submicron pores. Morphological change of the CA samples upon the SSI depended on the loaded amount of carvacrol. Low impregnation yield (6.0\%) resulted only in smoothening of the rugged surface. Higher load of carvacrol $(33.6 \%)$ led to the increased pores' diameter, while the complete collapse of the porous structure was observed for the maximum loading capacity of carvacrol (60\%) in CA beads (Figure 5). Similar observations were recently reported for CA impregnation with thymol [4, 5]. This phenomenon can be explained by participation of carvacrol with its hydroxyl group in intermolecular hydrogen bonds between CA chains leading to the change in solid structure of CA. Mallepally et al. [7] showed that loading of up to $25 \%$ of hydrogen peroxide did not affect morphology of CA mats. Shen et al. [8] also reported that loading of up to $10 \%$ of vanillin and L-menthol into CA fiber did not affect the morphology. Based on the results, it can be concluded that morphology changes of CA depend on the amount of impregnated carvacrol. The higher the carvacrol impregnation yield is, the more pronounced the change in CA will be. CA beads containing 6.0-33.6\% of carvacrol have a porous structure with submicron pores which is of interest for the controlled delivery applications [5].

3.3. Antibacterial Activity of Cellulose Acetate Samples. Antibacterial activity of the selected samples (impregnation yields of $6.0 \%, 33.6 \%$, and $60.0 \%$ ) and pure CA beads as control (C) against selected Gram-positive and Gramnegative bacterial strains is presented in Figure 6. As can 

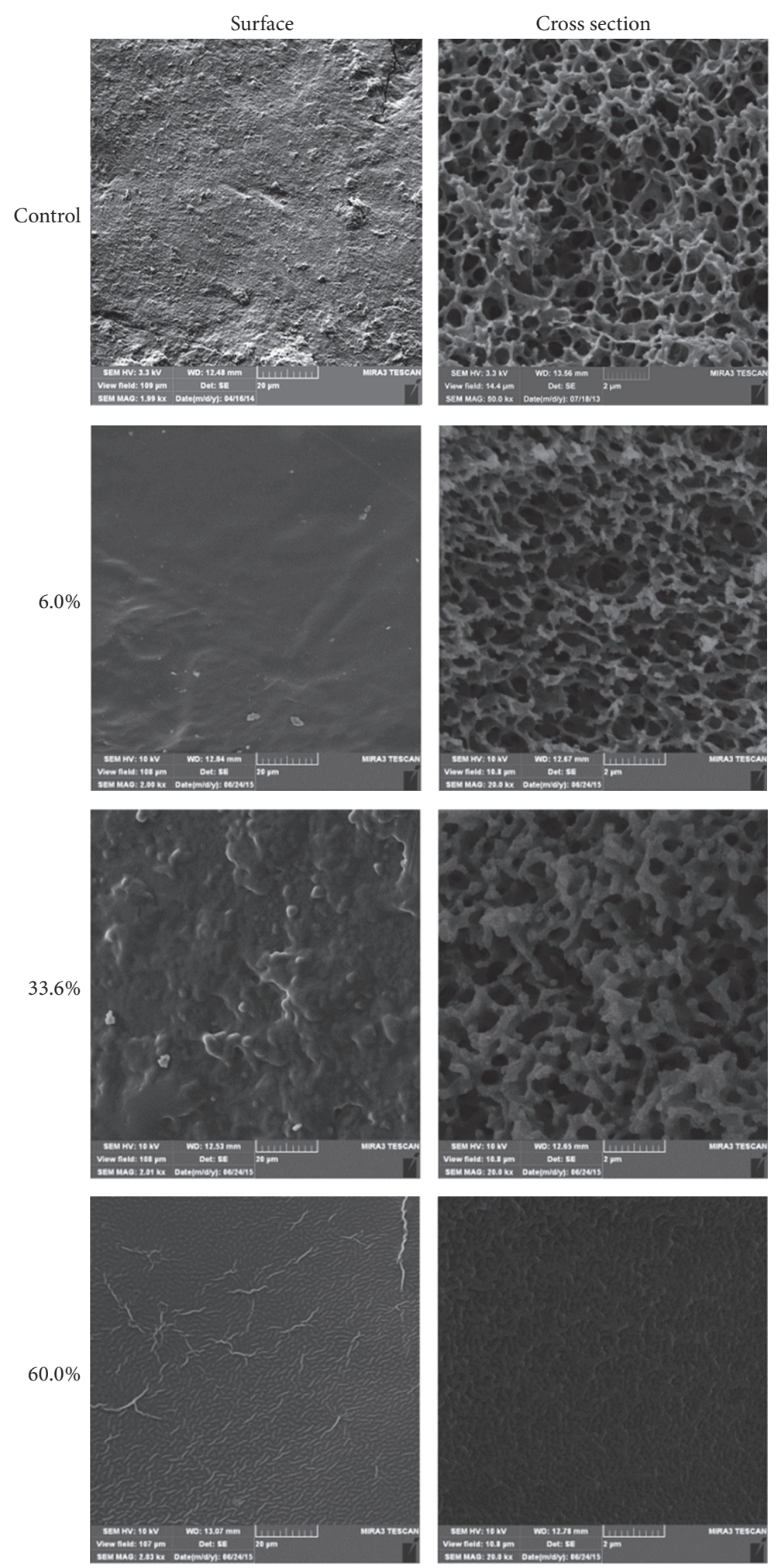

FIGURE 5: SEM images of cellulose acetate samples.

be seen, samples with carvacrol content of 33.6 and $60.0 \%$ showed considerable activity against all tested strains. Chosen bacterial strains, including foodborne pathogens and MRSA (methicillin-resistant S. aureus), are important in medical and veterinary clinical practice and are capable of causing different clinical disorders from banal, local infections to fatal systemic infection. Some of the investigated bacteria showed multiple antibiotic resistance. It was important to investigate 


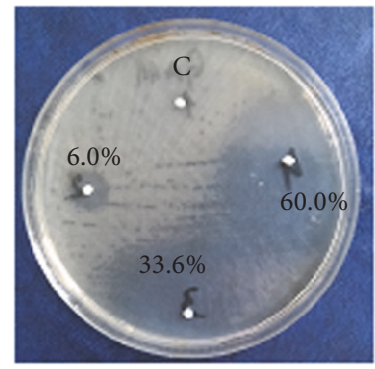

Acinetobacter sp.

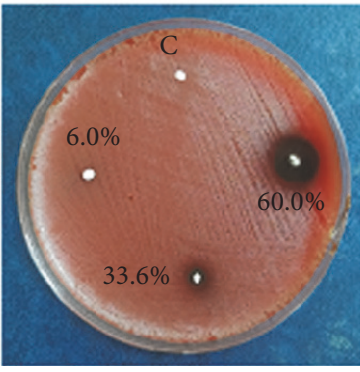

Corynebacterium sp.

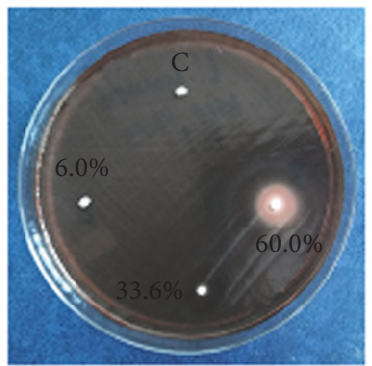

Listeria monocytogenes

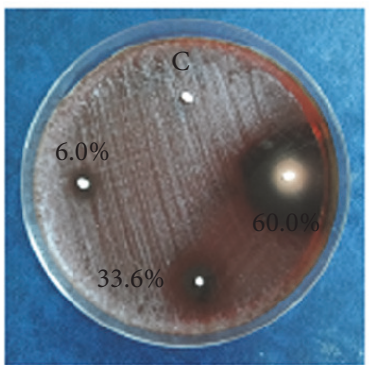

Rhodococcus equi

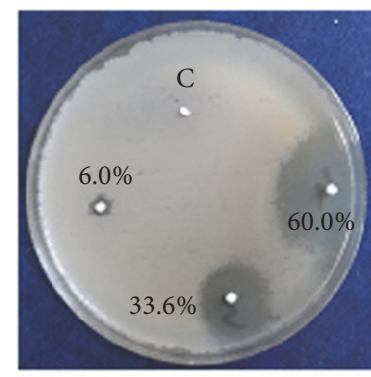

Bacillus anthracis

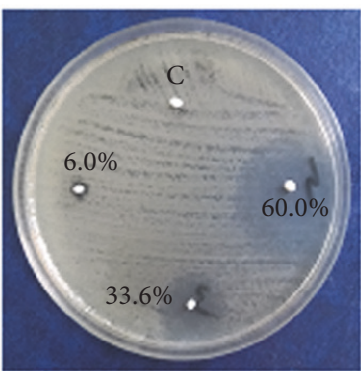

E. coli

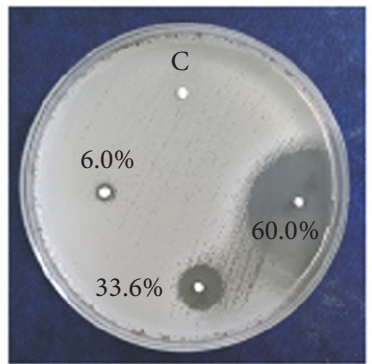

MRSA 7

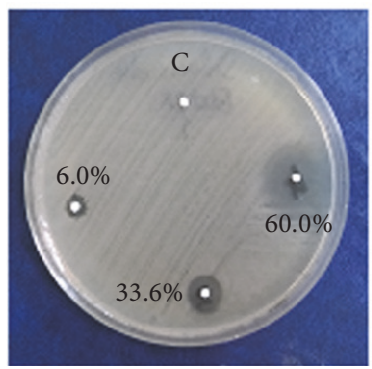

Salmonella Enteritidis

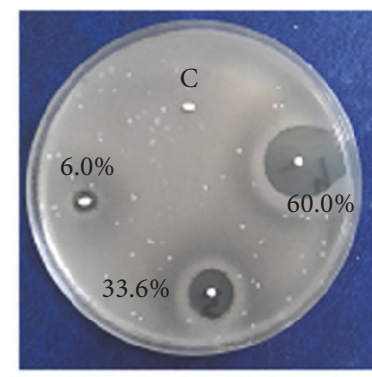

Bacillus cereus

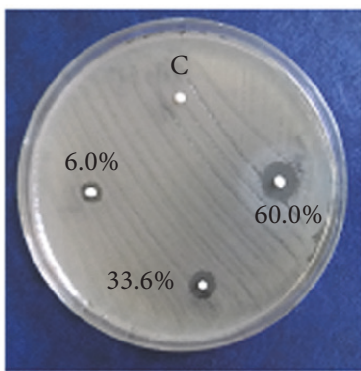

Klebsiella pneumonia

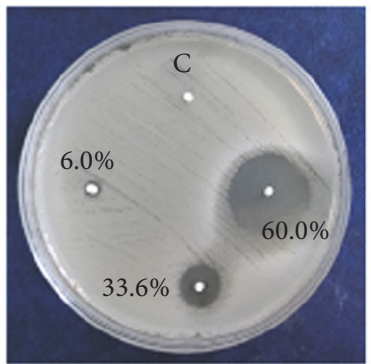

MRSA 74

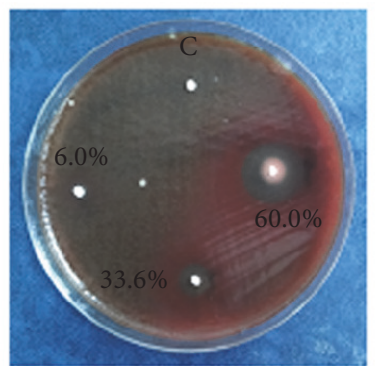

Streptococcus pneumonia

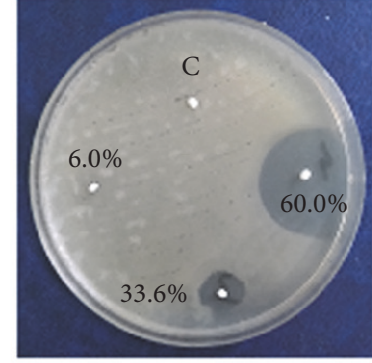

Bacillus subtilis

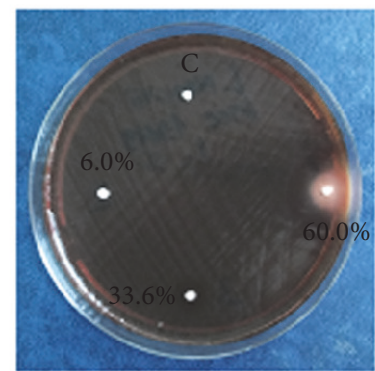

Listeria ivanovii

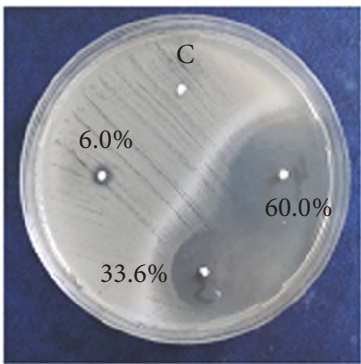

MRSA 75

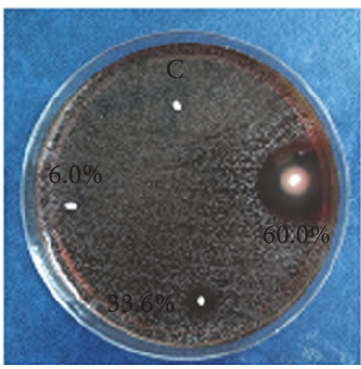

Streptococcus pyogenes

FIGURE 6: Inhibition zones of the tested samples.

if carvacrol was released from the CA on the surface of culture medium thus being capable of showing antibacterial activity. This is important for the possible implementation of the impregnated CA in clinical practice or in the food industry. Antimicrobial activity of a number of essential oils has been attributed to the presence of phenolic compounds. The inhibitory effect of phenols is explained by their interaction with the cell membrane of microorganisms [22]. Among phenolic compounds, carvacrol, an isoprenyl phenol, was reported to have one of the strongest antimicrobial activities [22]. Antimicrobial activity of carvacrol is sustained by its lipophilic character. It acts by disruption of the bacterial cytoplasmic membrane, which consequently loses its high impermeability resulting in bacteria's death [22].

Results of the qualitative assessment of the antibacterial activity of CA samples are presented in Table 2. Evidently, the control sample (pure CA) did not have any effect on the tested bacterial strains, while the impregnated samples showed diverse levels of antibacterial activity, from none to very strong (inhibition zones from 0 to $40 \mathrm{~mm}$ ). The strongest antibacterial activity was obtained with CA impregnated with $60 \%$ of carvacrol against all investigated strains except against K. pneumoniae and S. Enteritidis. Due to previously published results, it was not expected that CA $60 \%$ was going 
TABLE 2: Results of antibacterial analysis of pure CA and CA impregnated with carvacrol.

\begin{tabular}{|c|c|c|c|c|}
\hline \multirow{2}{*}{ Bacterial strain } & \multicolumn{4}{|c|}{ Inhibition zones (mm) } \\
\hline & Control & $6.0 \%$ & $33.6 \%$ & $60.0 \%$ \\
\hline Acinetobacter sp. (GA11) & 0 & 1 & 40 & 40 \\
\hline Bacillus anthracis & 0 & 5 & 16 & 32 \\
\hline Bacillus cereus & 0 & 6 & 12 & 20 \\
\hline Bacillus subtilis & 0 & 0 & 12 & 23 \\
\hline Corynebacterium sp. & 0 & 0 & 7 & 15 \\
\hline Escherichia coli & 0 & 4 & 14 & 20 \\
\hline Klebsiella pneumoniae (GA15) & 0 & 6 & 7 & 10 \\
\hline Listeria monocytogenes ATCC19111 & 0 & 0 & 12 & 20 \\
\hline Listeria ivanovii ATCC19119 & 0 & 0 & 6 & 21 \\
\hline Methicillin-resistant S. aureus (MRSA 7) & 0 & 4 & 12 & 30 \\
\hline Methicillin-resistant $S$. aureus (MRSA 74) & 0 & 4 & 11 & 22 \\
\hline Methicillin-resistant S. aureus (MRSA 75) & 0 & 5 & 20 & 30 \\
\hline Rhodococcus equi & 0 & 6 & 14 & 30 \\
\hline Salmonella Enteritidis & 0 & 5 & 9 & 19 \\
\hline Streptococcus pyogenes & 0 & 0 & 9 & 21 \\
\hline Streptococcus pneumoniae & 0 & 10 & 23 & 35 \\
\hline
\end{tabular}

to show some significant activity against Gram-negative strains. Anyway, strong inhibition zone of CA $60 \%$ around E. coli and Acinetobacter indicates visible susceptibility of these particular strains to carvacrol so further investigations on this subject are needed meaning not all Gram-negative strains are equally susceptible/resistant to carvacrol. Susceptibility of Gram-negative bacterial strains to carvacrol is obviously dependent on some metabolic mechanisms of investigated bacteria which need to be further investigated. Obtained results of strong activity of the CA impregnated with carvacrol could bring possible solution to treatment of patients with wounds infected with Acinetobacter especially with strains resistant to carbapenems. Carbapenem-resistant Acinetobacter are placed on the World Health Organization priority pathogens list and are marked as Priority 1, critical [32]. Small inhibition zone (weak activity) of CA 60\% against $S$. pyogenes is strange considering the fact that carvacrol like many other bioactive compounds obtained from plants has strong activity against Gram-positive bacteria. Strong antibacterial activity of CA $60 \%$ against Listeria strains indicates possible implementation of impregnated CA in food industry as a protective agent against multiplication of these foodborne pathogens, especially in fish industry. CA $60 \%$ could also be used as the protection against food spoilage caused by Bacillus spp. The CA sample with the lowest carvacrol content $(6 \%)$ had weak to moderate antibacterial activity. Our previous study showed that lower percentage of thymol in CA $(4.5 \%)$ had no bacterial effect, while the CA containing $13.7 \%$ of thymol had significant antibacterial activity against Salmonella Typhimurium, Salmonella Enteritidis, Listeria monocytogenes, Listeria ivanovii, Listeria innocua, Corynebacterium spp., Rhodococcus equi, Bacillus anthracis, Bacillus cereus, Bacillus subtilis, Streptococcus pneumoniae, Streptococcus pyogenes, Staphylococcus aureus MRSA ATCC 33591, clinical isolates of methicillin-resistant
S. aureus, Klebsiella pneumoniae, Pseudomonas aeruginosa, Escherichia coli, Acinetobacter sp., and Proteus mirabilis [5].

The samples with carvacrol impregnation yield of $33.6 \%$ and $60.0 \%$ showed considerable antibacterial activity against all the tested strains which means that the amount of carvacrol released from the CA was sufficiently high to reach the critical concentration for the inhibition of bacterial growth. Particularly important result is the antibacterial activity against MRSA strains bearing in mind the problem of bacteria's multiresistance to antibiotics, whereby even topical infections (skin, eye, or ear) caused by MRSA may have fatal outcome [33].

\section{Conclusion}

The results from this study showed that CA in the form of beads with submicron pores can be successfully impregnated with carvacrol using supercritical impregnation process with $\mathrm{CO}_{2}$. Results also showed that carvacrol impregnation yield increased with the increase of operating pressure and time. The impregnation was faster when conducted at higher pressures due to the higher solubility of carvacrol in $\mathrm{scCO}_{2}$. The maximum carvacrol impregnation yield into $\mathrm{CA}$ was found to be around $60 \%$. Impregnation yields higher than $6 \%$ lead to morphological changes in CA from swelling to the complete loss of pores $(60 \%)$ due to the interference of carvacrol with intermolecular hydrogen bonds between the CA chains. Impregnated samples with higher impregnation yield $(33.6 \%$ and $60.0 \%)$ showed considerable antibacterial activity against selected Gram-positive and Gram-negative bacterial strains including MRSA, in contrast to pure CA which did not show any antibacterial activity.

According to the presented results, SSI at pressure of $30 \mathrm{MPa}$ and temperature of $50^{\circ} \mathrm{C}$ during $2 \mathrm{~h}$ is suggested for production of porous CA beads containing around $36 \%$ of 
carvacrol which was proven to be sufficient for antibacterial effect against chosen bacterial strains including MRSA.

Simple tuning of the SSI operating conditions enables manipulation of the additive impregnation yield and allows production of CA with desired carvacrol content for targeted antibacterial application. The supercritical impregnation process using $\mathrm{CO}_{2}$ was shown to be a feasible technique for fabrication of CA impregnated with carvacrol for different possible application when solvent free and biodegradable material with antibacterial properties is required (e.g., food and pharmaceutical industry as well in medicine). In further studies it is necessary to investigate release kinetics of carvacrol from the impregnated CA samples in surrounding media of interest.

\section{Abbreviations}

CA: Cellulose acetate

MRSA: Methicillin-resistant Staphylococcus aureus

SSI: Supercritical solvent impregnation

$\mathrm{scCO}_{2}$ : Supercritical carbon dioxide.

\section{Disclosure}

Results of this study were presented at 15th European Meeting on Supercritical Fluids (May 8-11, 2016, Essen, Germany).

\section{Conflicts of Interest}

The authors declare that there are no conflicts of interest regarding the publication of this paper.

\section{Acknowledgments}

Financial support of this work from the Ministry of Education, Science and Technological Development of the Republic of Serbia (Project III 45017) is gratefully acknowledged. Generous donation of the cellulose acetate from Dr. Maciej Posel from Eastman Chemical, Poland, is appreciated.

\section{References}

[1] S. G. Kazarian, "Polymer processing with supercritical fluids," Polymer Science, vol. 42, no. 1, pp. 78-101, 2000.

[2] S. Milovanovic, I. Jankovic-Castvan, J. Ivanovic, and I. Zizovic, "Effect of starch xero- And aerogels preparation on the supercritical $\mathrm{CO}_{2}$ impregnation of thymol," Starch/Stärke, vol. 67, no. 1-2, pp. 174-182, 2015.

[3] S. Milovanovic, M. Stamenic, D. Markovic, M. Radetic, and I. Zizovic, "Solubility of thymol in supercritical carbon dioxide and its impregnation on cotton gauze," Journal of Supercritical Fluids, vol. 84, pp. 173-181, 2013.

[4] S. Milovanovic, M. Stamenic, D. Markovic, J. Ivanovic, and I. Zizovic, "Supercritical impregnation of cellulose acetate with thymol," The Journal of Supercritical Fluids, vol. 97, pp. 107-115, 2015.

[5] S. Milovanovic, D. Markovic, K. Aksentijevic, D. B. Stojanovic, J. Ivanovic, and I. Zizovic, "Application of cellulose acetate for controlled release of thymol," Carbohydrate Polymers, vol. 147, pp. 344-353, 2016.

[6] J. Ivanovic, S. Knauer, A. Fanovich et al., "Supercritical $\mathrm{CO}_{2}$ sorption kinetics and thymol impregnation of PCL and PCLHA," Journal of Supercritical Fluids, vol. 107, pp. 486-498, 2016.

[7] R. R. Mallepally, M. A. Marin, N. Montesdeoca, C. Parrish, K. R. Ward, and M. A. McHugh, "Hydrogen peroxide loaded cellulose acetate mats as controlled topical $\mathrm{O}_{2}$ delivery devices," Journal of Supercritical Fluids, 2014.

[8] Z. Shen, G. S. Huvard, C. S. Warriner, M. Mc Hugh, J. L. Banyasz, and M. K. Mishra, "CO2-assisted fiber impregnation," Polymer, vol. 49, no. 6, pp. 1579-1586, 2008.

[9] A. M. A. Dias, M. E. M. Braga, I. J. Seabra, P. Ferreira, M. H. Gil, and H. C. De Sousa, "Development of natural-based wound dressings impregnated with bioactive compounds and using supercritical carbon dioxide," International Journal of Pharmaceutics, vol. 408, no. 1-2, pp. 9-19, 2011.

[10] N. Lavoine, C. Givord, N. Tabary, I. Desloges, B. Martel, and J. Bras, "Elaboration of a new antibacterial bio-nano-material for food-packaging by synergistic action of cyclodextrin and microfibrillated cellulose," Innovative Food Science and Emerging Technologies, vol. 26, pp. 330-340, 2014.

[11] G. Kavoosi, S. M. M. Dadfar, A. Mohammadi Purfard, and R. Mehrabi, "Antioxidant and antibacterial properties of gelatin films incorporated with carvacrol," Journal of Food Safety, vol. 33, no. 4, pp. 423-432, 2013.

[12] Y. Yao, D. Ding, H. Shao, Q. Peng, and Y. Huang, "Antibacterial Activity and Physical Properties of Fish Gelatin-Chitosan Edible Films Supplemented with D-Limonene," International Journal of Polymer Science, vol. 2017, Article ID 1837171, 9 pages, 2017.

[13] M. Pantić, P. Kotnik, Ž. Knez, and Z. Novak, "High pressure impregnation of vitamin D3 into polysaccharide aerogels using moderate and low temperatures," Journal of Supercritical Fluids, vol. 118, pp. 171-177, 2016.

[14] S. Cardea, M. Scognamiglio, and E. Reverchon, "Supercritical fluid assisted process for the generation of cellulose acetate loaded structures, potentially useful for tissue engineering applications," Materials Science and Engineering C, vol. 59, pp. 480-487, 2016.

[15] P. Marizza, L. Pontoni, T. Rindzevicius et al., "Supercritical impregnation of polymer matrices spatially confined in microcontainers for oral drug delivery: effect of temperature, pressure and time," Journal of Supercritical Fluids, vol. 107, pp. 145-152, 2016.

[16] P. L. Beltrame, Castelli, E. Selli et al., "Dyeing of cotton in supercritical carbon dioxide," Dyes and Pigments, vol. 39, no. 4, pp. 335-340, 1998.

[17] A. S. Özcan, A. A. Clifford, K. D. Bartle, and D. M. Lewis, "Dyeing of cotton fibres with disperse dyes in supercritical carbon dioxide," Dyes and Pigments, vol. 36, no. 2, pp. 103-110, 1998.

[18] S. Burt, "Essential oils: their antibacterial properties and potential applications in foods-a review," International Journal of Food Microbiology, vol. 94, no. 3, pp. 223-253, 2004.

[19] S. G. Deans and G. Ritchie, "Antibacterial properties of plant essential oils," International Journal of Food Microbiology, vol. 5, no. 2, pp. 165-180, 1987.

[20] J. M. Jay and G. M. Rivers, "Antimicrobial activity of some food flavoring compounds," Journal of Food Safety, vol. 6, no. 2, pp. 129-139, 1984. 
[21] G. A. Leeke, R. Santos, and M. B. King, "Vapor-liquid equilibria for the carbon dioxide + carvacrol system at elevated pressures," Journal of Chemical and Engineering Data, vol. 46, no. 3, pp. 541545, 2001.

[22] A. Ben Arfa, S. Combes, L. Preziosi-Belloy, N. Gontard, and P. Chalier, "Antimicrobial activity of carvacrol related to its chemical structure," Letters in Applied Microbiology, vol. 43, no. 2, pp. 149-154, 2006.

[23] C. C. Liolios, O. Gortzi, S. Lalas, J. Tsaknis, and I. Chinou, "Liposomal incorporation of carvacrol and thymol isolated from the essential oil of Origanum dictamnus L. and in vitro antimicrobial activity," Food Chemistry, vol. 112, no. 1, pp. 7783, 2009.

[24] L. Keawchaoon and R. Yoksan, "Preparation, characterization and in vitro release study of carvacrol-loaded chitosan nanoparticles," Colloids and Surfaces B: Biointerfaces, vol. 84, no. 1, pp. 163-171, 2011.

[25] E. F. Evans and L. F. McBurney, "Heat stability of cellulose acetate," Industrial \& Engineering Chemistry, vol. 41, no. 6, pp. 1260-1264, 1949.

[26] A. Bernal-Ballén, I. Kuritka, and P. Saha, "Preparation and characterization of a bioartificial polymeric material: bilayer of cellulose acetate-PVA," International Journal of Polymer Science, vol. 2016, Article ID 3172545, 2016.

[27] Y. Kurokawa and K. Hanaya, "Functionality of cellulose by impregnation of inorganic substances," Carbohydrate Polymers, vol. 27, no. 4, pp. 313-320, 1995.

[28] F.-J. Wang, Y.-Y. Yang, X.-Z. Zhang, X. Zhu, T.-S. Chung, and S. Moochhala, "Cellulose acetate membranes for transdermal delivery of scopolamine base," Materials Science and Engineering C, vol. 20, no. 1-2, pp. 93-100, 2002.

[29] G. d. Dannenberg, G. D. Funck, C. E. Cruxen, J. d. Marques, W. P. Silva, and Â. M. Fiorentini, "Essential oil from pink pepper as an antimicrobial component in cellulose acetate film: potential for application as active packaging for sliced cheese," $L W T$ Food Science and Technology, vol. 81, pp. 314-318, 2017.

[30] M. M. Castillo-Ortega, A. Nájera-Luna, D. E. Rodríguez-Félix et al., "Preparation, characterization and release of amoxicillin from cellulose acetate and poly(vinyl pyrrolidone) coaxial electrospun fibrous membranes," Materials Science and Engineering C, vol. 31, no. 8, pp. 1772-1778, 2011.

[31] H. D. Isenberg, Detection of methicillin resistance in Staphylococci by PCR. 12.5.3.1, Clinical Microbiology Procedures Handbook, ASM Press, Wash, USA, 2nd edition, 2004.

[32] 2007, http://www.who.int/medicines/publications/WHO-PPLShort_Summary_25Feb-ET_NM_WHO.pdf.

[33] R. M. Klevens, M. A. Morrison, J. Nadle et al., "Invasive methicillin-resistant Staphylococcus aureus infections in the United States," Journal of the American Medical Association, vol. 298, no. 15, pp. 1763-1771, 2007. 

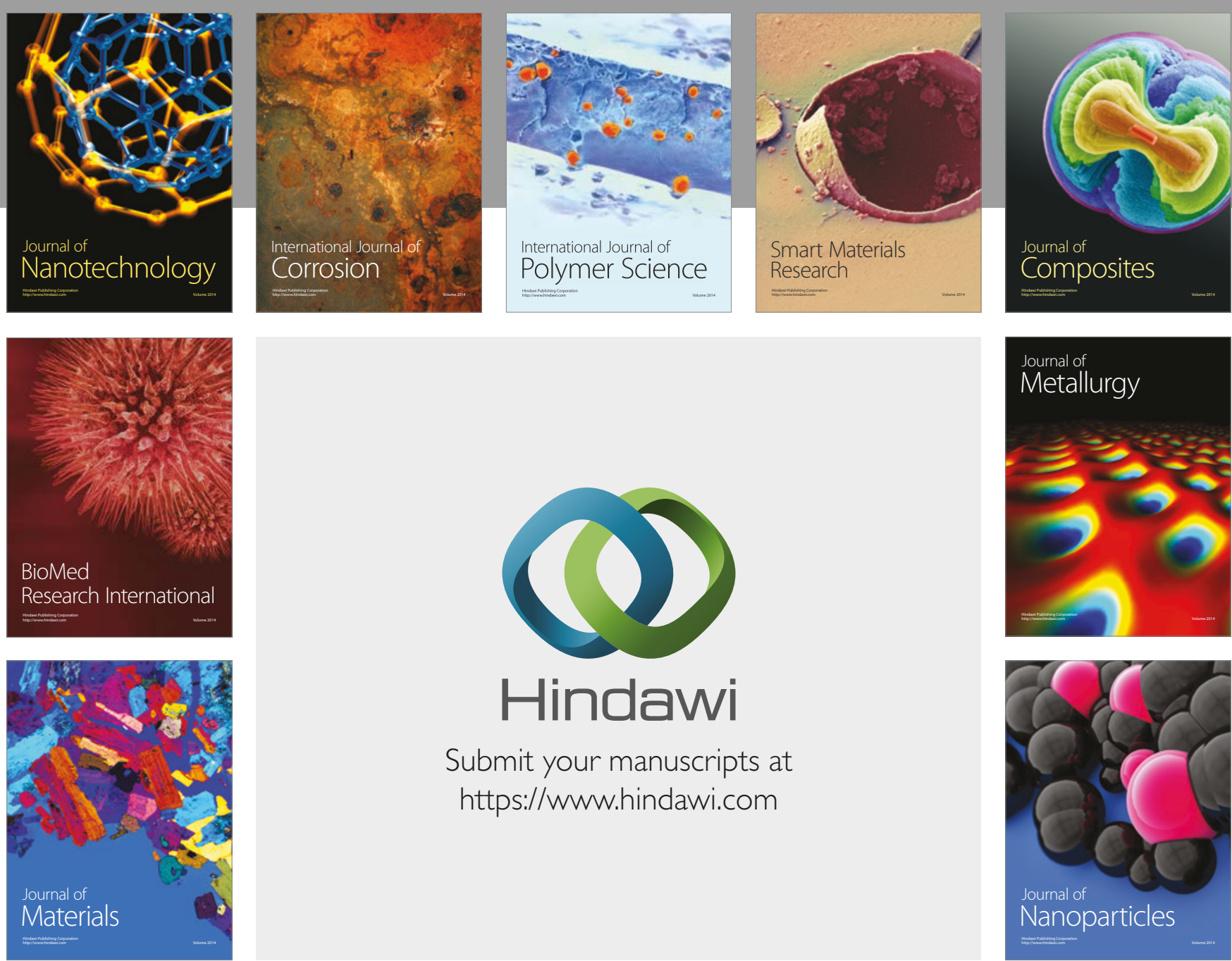

\section{Hindawi}

Submit your manuscripts at

https://www.hindawi.com
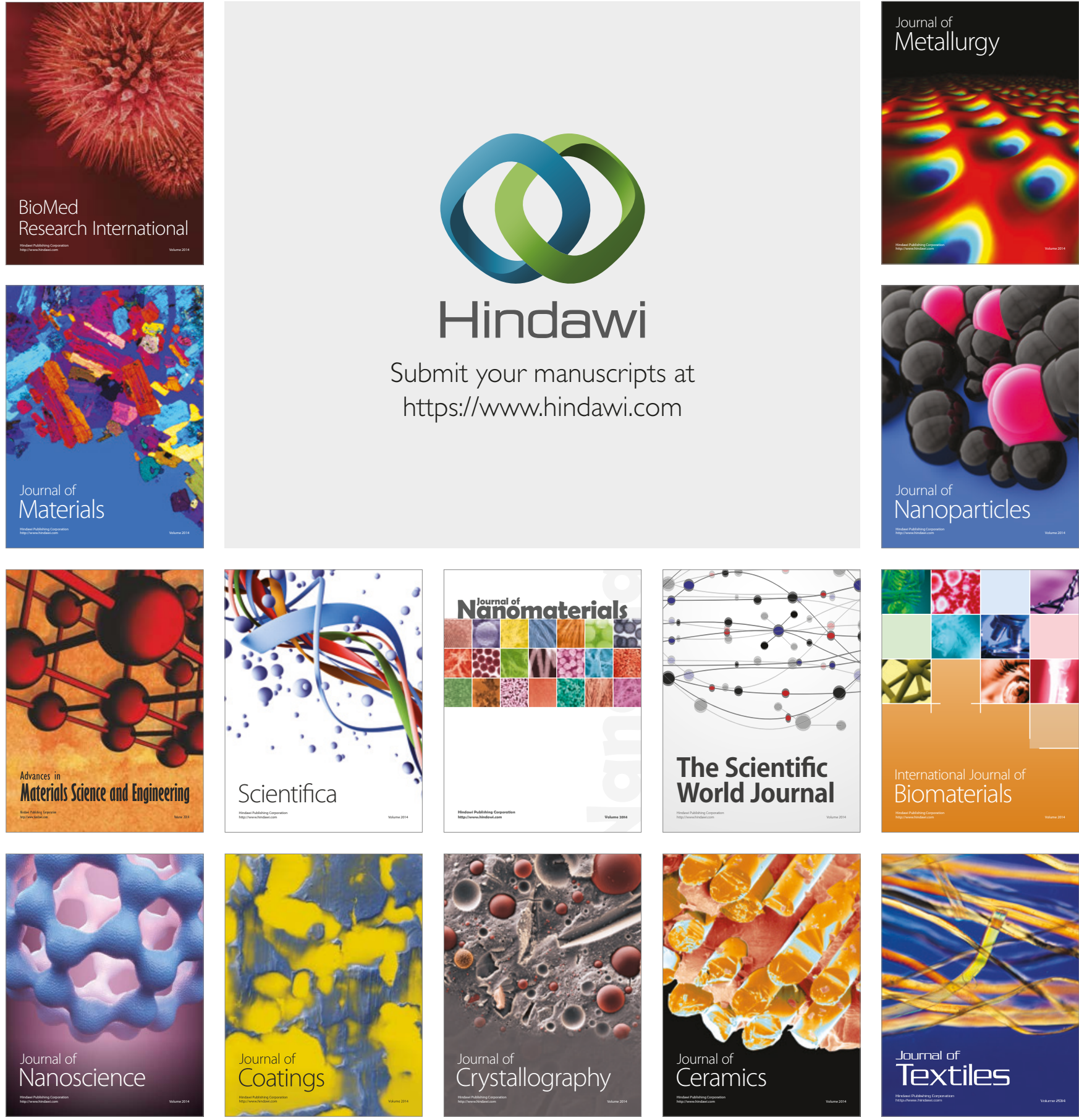

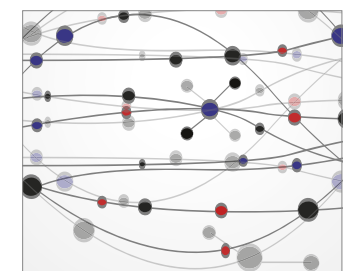

The Scientific World Journal
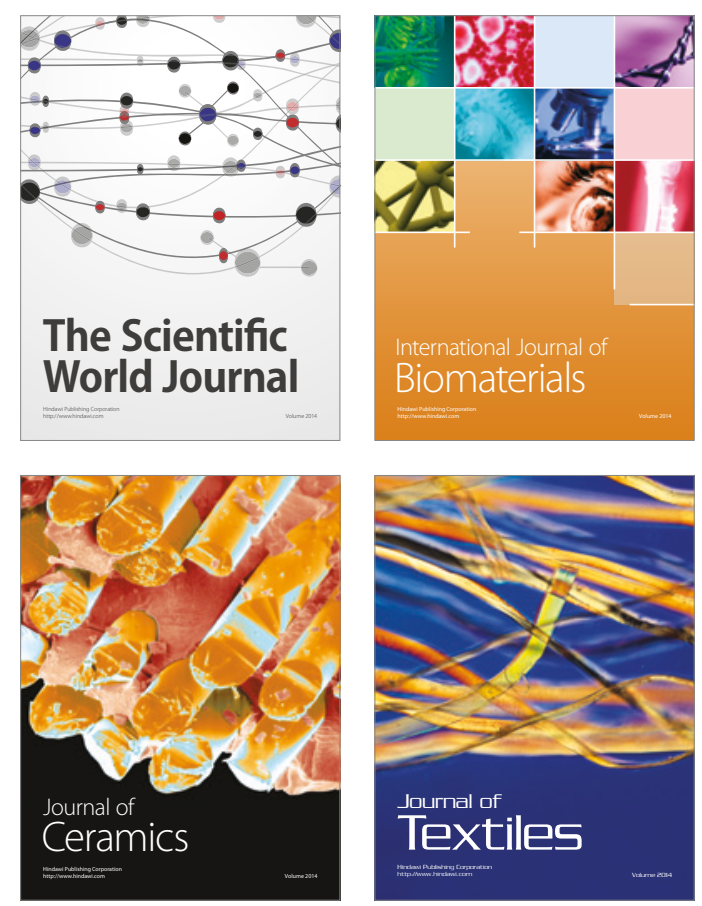